\title{
Challenging Inferior Vena Cava Leiomyosarcoma: A Case Report and Literature Review of Management
}

\author{
Homoud AlAwfi ${ }^{1}$, Huda S. AlMaghrabi ${ }^{1}$, Faisal H. AlSarrani ${ }^{1}$, Mohammad A. \\ Alzahrani* \\ Department of Surgery, King Abdulaziz Medical City, Riyadh
}

\begin{abstract}
Received: 01 August, 2016; Accepted: 16 August, 2016; Published: 26 August, 2016
*Corresponding author: Mohammed A Alzahrani, Consultant Surgical Oncologist and Endocrine Surgeon, Department of Surgery, Ext 15548, King Abdulaziz Medical City, Riyadh, Tel: 009661801 1111; Email: zahranimo@NGHA.MED.SA
\end{abstract}

\begin{abstract}
Jim Abstract
Leiomyosarcoma (LMS) of the inferior vena cava (IVC) is a rare entity. Clinical presentation is largely size dependent and the mainstay of treatment is radical margin negative resection. Surgical treatment can be challenging depending on the anatomical location of the LMS in the IVC. Due to high incidence of recurrence, other treatment components including chemotherapy and radiation are frequently included in the overall treatment strategy. We here present a case of 23-pound IVC LMS diagnosed in a 53 year-old female. To the best of our knowledge, this is the largest IVC LMS published in the literature. She had radical resection that entailed an En Block radical resection of the sarcoma, right kidney and infra-renal IVC. At one year follow up, she is still enjoying disease free hiatus.
\end{abstract}

Keywords: Leiomyosarcoma; Sarcoma; Smooth muscle tumor; Inferior vena cava

\section{Introduction}

Primary tumors of the Inferior Vena Cava (IVC) are rare, with Leiomyosarcoma (LMS) representing the vast majority (95\%). Greater than $50 \%$ of all vascular LMSs occur in the IVC. Prognosis is often guarded due to advanced stage at diagnosis. Surgical resection of the tumor and affected segment of IVC with negative margins is the only treatment shown to improve survival; the use of neoadjuvent chemotherapy alone or combined with radiation therapy is still controversial due to lack of large patient cohorts.

\section{Case Report}

A 53 years old lady presented to the clinic with a complaint of abdominal pain and nausea for 2 months. The patient denied fevers, chills, vomiting, hematuria, dysuria or weight loss. Her past medical history was consistent with hypertension, osteoarthritis and iron deficiency anemia. Examination revealed a soft lax abdomen with no organomegaly or mass that can be felt. Her laboratory investigations, including hematologic panel, chemistry, and liver function tests, were found to be normal, apart from mild hypochromic microcytic anemia (Hgb $11.2 \mathrm{~g} / \mathrm{dl}$ ).

Her initial treating physician was suspecting gall bladder disease so an Ultrasound (US) was done which demonstrated no abnormality. It however revealed an incidental finding of 10 * $5 \mathrm{~cm}$ mass in the right pararenal area. Subsequently, a CT scan was done which revealed a $10.8 * 7.5 * 6.1 \mathrm{~cm}$ right "Para-renal" mass with compression of inferior vena cava and moderate right hydronephrosis, (Figure 1, 2). Abdomen MRI shows $11 * 6 * 8$ $\mathrm{cm}$ with high signal intensity and adherent to the IVC. The initial impression of her treating physician was that of an adrenal tumor.

Full endocrine biochemical evaluation was done and was completely normal, so discussion for surgical resection was carried out but the patient refused and she lost fellow up.

Three years later, she presented again to the clinic complaining

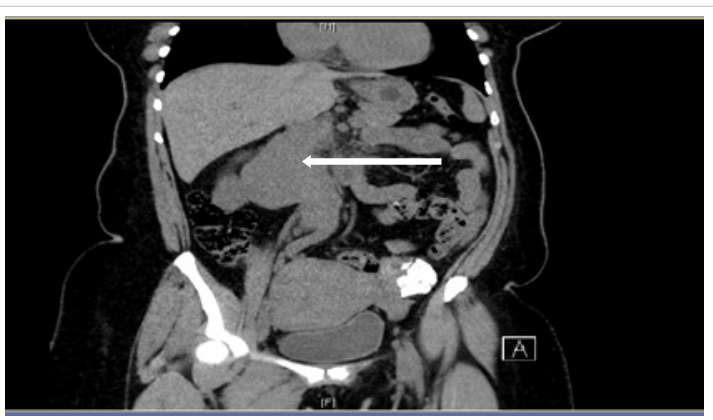

Figure 1: CT Abdomen showing part of the Pararenal mass.

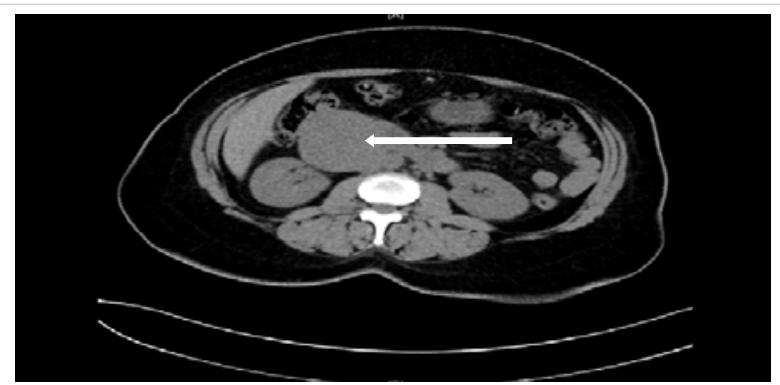

Figure 2: Axial view of the mass with its vascular relations. 
of increasing abdominal pain and girth. On physical examination, she clinically had a large $15 * 20 \mathrm{~cm}$ abdominal mass.

High resolution CT demonstrated $18 * 19 * 20 \mathrm{~cm}$ in the right pararenal region with compression of IVC and moderate hydronephrosis, there was no evidence of metastasis to the brain or lungs (Figure 3,4). Nuclear Renal Scan showed nonfunctioning right kidney. Tru-cut biopsy result was compatible withLMS. The specimen was inadequate to characterize grade although remarkable degree of necrosis was perceived as a surrogate of high-grade pathology.

The case was discussed at a multidisciplinary tumor board and the decision was to start a trial of neoadjuvent chemotherapy (ifosfomide and doxorubicin). Repeated images after a couple of cycles showed no significant radiographic response to chemotherapy by RECIST criteria, so the decision was to go for En Block surgical resection.

Intraoperatively, $25 * 18 \mathrm{~cm}$ tumor occupying the whole right side of the abdomen adherent to the duodenum, small bowel (Figure 5, 6) and direct invasion of the infrarenal IVC portion and right kidney. Initially, careful, mostly sharp, mobilization of the right colon and the root of mesentry (Cattell - Braasch Maneuver) Due to the retroperitoneal location of the tumor, duodenum, colon, and small bowel loops can be overstretched over the mass and this warrants cautious sharp dissection and gentle handling in order to avoid inadvertent injury to those structures. The IVC was isolated with proximal and distal control where caval dissection was continued cephalad to the retrohepatic portion and caudal to the iliac veins. After exposing and safe guarding the left renal vein, we used vascular staples to transect through infrarenal IVC leaving $1 \mathrm{~cm}$ margin away from the tumor. The specimen was removed En-block with the right kidney and, infrarenal IVC. Due to the fact that tumor was primarily infrarenal and that the tumor was compressing the IVC for prolonged time, we encountered significant collateral neo-angiogenesis. Therefore, we elected to abandon reconstructing the IVC.

Her postoperative course was smooth; she spent three nights in the ICU and then shifted to the surgical floor where she spent 4 more days before she was discharged home.

Review of the pathology specimens demonstrated a $27 \mathrm{~cm}$ tumor; weight 22.5 pounds consisting of LMS with $50 \%$ necrosis, French Federation Nationale des Centers de Lutte Contre le Cancer (FNCLCC) histologic grade 3. The tumor originated from the IVC at the origin of right renal vein, No lymphovascular invasion. Four Lymph nodes were identified and were negative, All surgical resection margins were negative.

Immunohistochemistry:

$\begin{array}{ll}\text { SMA (1A4): } & \text { Positive } \\ \text { DESMIN (D33): } & \text { Positive } \\ \text { Caldesmon(H-CD): } & \text { Positive } \\ \text { Vimentin (V9): } & \text { Positive } \\ \text { S100 (polyclonal): } & \text { Negative } \\ \text { HMB 45: } & \text { Negative } \\ \text { CK pan (AE1/AE3): } & \text { Negative }\end{array}$

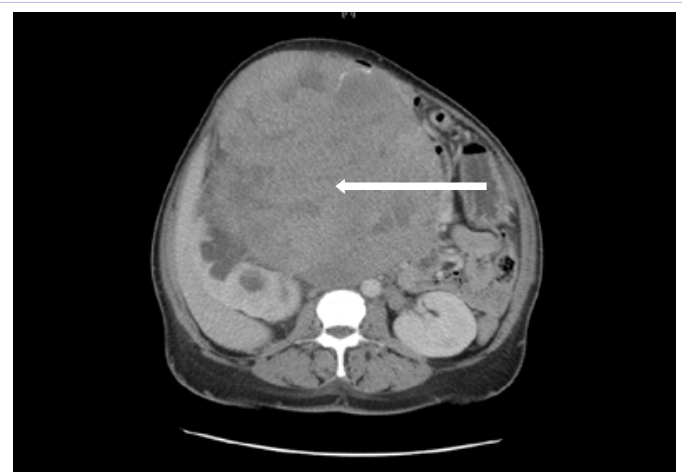

Figure 3: Axial view of the mass with its vascular relations.

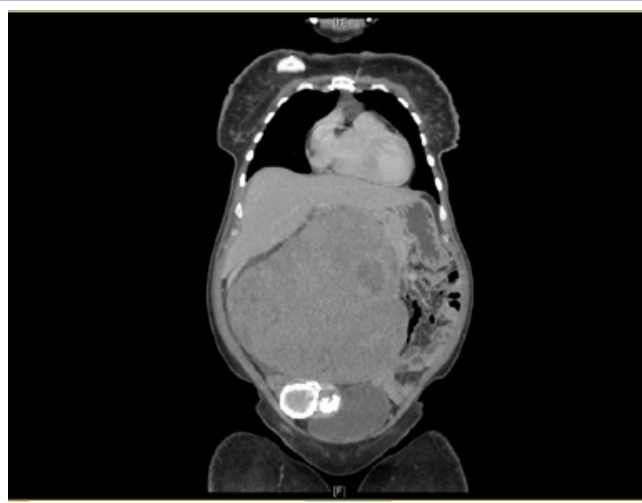

Figure 4: Another view of the Mass.

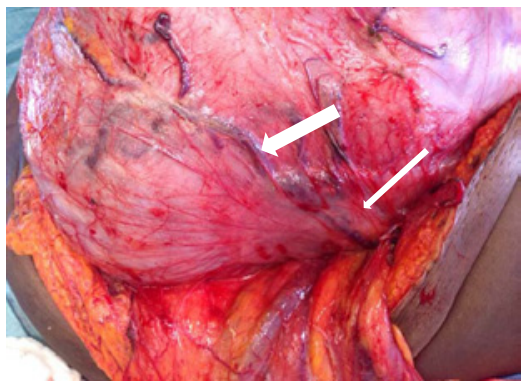

Figure 5: Intra op, small arrow Duodenum, Large arrow one of the feeding vessels.

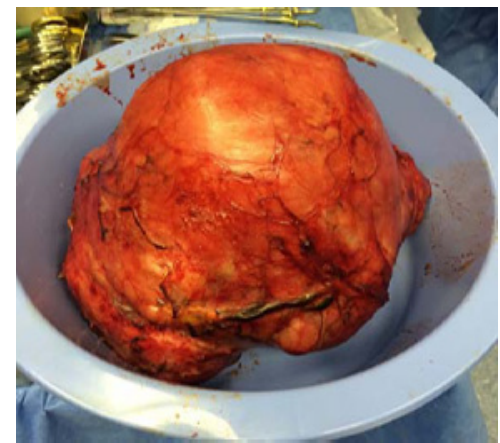

Figure 6: The mass was removed En Block (Right Kidney \& segment of the IVC). 
The case was further discussed at the multidisciplinary tumor board to address the potential value of adjuvant chemotherapy and/or radiotherapy. Due to successful R0 resection, the potential of radiation toxicity given large field, and lack of significant response to the neoadjevant chemotherapy trial, the decision was not to give any form of adjuvant therapy and to keep the patient on a strict follow up to detect any recurrence. Until writing this manuscript; our patient completed 22 months with no radiographic sign of local or distant failure.

\section{Discussion}

Leiomyosarcoma of the IVC is extremely rare, and the incidence ranges between 0.5 to $1 \%$ of all sarcomas. Being that rare, available evidence that is used to guide management and follow up is mostly in the form of case reports and case series [1].

Primary IVC LMS represents a management challenge. Consistent with other published reports [1], this report demonstrates that surgical resection can be safely performed and is associated with improved survival.

The three most common presenting symptoms are abdominal pain (52\%), distention (20\%), and deep venous thrombosis (12\%) [1]. Other symptoms include Budd-Chiari syndrome, weight loss, fever, weakness, anorexia, vomiting, night sweats, and dyspnea.

Leiomyosarcomas tend to enlarge via intraluminal or extraluminal growth rather than in filtration [1].Imaging usually demonstrates extraluminal tumor in the retroperitoneum. If they involve adjacent organs, these tumors are often mistaken for primary LMS arising from retroperitoneum rather than the IVC.

However, such a tumor should be classified as a LMS of the IVC if it has predominantly intraluminal growth or if a segment of the involved IVC needs to be resected with the extraluminal tumor. Retroperitoneal LMS is a rare entity; accounting for 3-5\% of all retroperitoneal tumors [2].

Leiomyosarcoma of the inferior vena cava typically arises from the smooth muscle lining the wall of the infra-renal (44.2\%), middle portion (trans-renal to retrohepatic IVC) (50.8\%), or the upper (suprahepatic) portion of the IVC (4.2\%) [3]. Given their location deep in the retroperitoneum and the slow growth, these tumors usually have an insidious presentation [4]; they are most frequently seen in the sixth decade with a female predominance with symptoms occurring at late stages of the disease.

In 1992, the International Registry of Inferior Vena Cava LMS collected 218 cases. In that series, tumors arose from the IVC lower segment in 80 patients, from the middle in 94 , and from the upper IVC in 41 [5]. IVC LMS arising below the renal vein (segment I) may cause pain in the right-lower quadrant, back, and flank as well as varying degrees of lower extremity edema. Those arising in the middle caval segment (segment II) may cause right-upper-quadrant pain and occasionally renovascular hypertension. Those arising above the hepatic vein (segment III) may cause varying manifestations of the Budd-Chiari syndrome [6].
Surgical resection with negative margin for the localized tumors remains the standard potentially curative treatment for patients with retroperitoneal sarcoma. Margin status is the most contributing factor for long term Disease Free Survival (DFS) [7].

A series of 500 patients in a single institute reported a median survival of 103 months for those who underwent R0 resection in contrast to 18 months for those with incomplete resection [8].

Local control was consistently reported to improve with aggressive surgical approach in retrospective analyses $[9,10]$.

As noted earlier, there is paucity of robust data on neoadjuvent treatment of IVC LMS in particular; this strategy has been attempted in other retroperitoneal sarcomas. The argument for this strategy was to attempt downsizing the tumor and hence increasing resectability rates. Additionally, preoperative Radiation Therapy (RT) might increase the chances to achieve R0 resection and decrease the risk of tumor seeding during surgical manipulation [11].

Pawlik and his colleagues reported two prospective studies showing a favorable 5 year local Relapse Free Survival (RFS) (60\%), DFS (46\%), and OS (61\%) following an R0 or R1 resection after preoperative RT in patients with intermediate or high grade retroperitoneal sarcoma [12].

The role of pre-Vs. postoperative chemotherapy for IVC LMS is not clearly defined in published data for retroperitoneal sarcoma, in contrast to the more clear benefits reported for extremity and other trunk Soft Tissue Sarcoma (STS).

The reported studies for preoperative chemotherapy showed inconsistent findings, one randomized study reported no survival benefits for patients receiving chemotherapy preoperatively vs. surgery alone $[13,14,15]$.

Postoperative RT was recommended by NCCN guidelines for highly selective patients with incomplete resection, high-grade tumors or high risk of recurrence. On the other hand, patients with R0 resection might receive RT if the toxicity to the adjacent normal structures can be avoided or minimized.

The largest literature review of IVC LMS to date reported 218 patients, 5- and 10-year survival rates of $49.4 \%$ and $29.5 \%$, respectively [16]. Given the proximity of the middle section of the IVC to numerous retroperitoneal organs and the extensive innervation in that area, tumors involving this portion of the IVC generally are symptomatic earlier and more often. Because of this earlier presentation, these tumors generally have a better prognosis, with 5 - and 10 -year survival rates of $56.7 \%$ and $47.3 \%$ $[16,17]$.

Recently in 2015 Sulpice and his colleges reported a series of 8 cases of LMs of IVC between 1997-2013 their conclusion was similar to most of the previous reported case as the main therapeutic option is R0 resection with or without IVC construction based on the location of the tumor, in case of recurrence surgery with $\mathrm{R} 0$ resection remain the main treatment option [18]. 
Inferior vena cava leiomyosarcoma has been reported to have a comparable outcome with other leiomyosarcomas arising when matched for size. Long-term survival is possible for patients with localized IVC leiomyosarcomas that are completely resected, and it appears that radiation therapy improves outcomes. The exact role of radiation therapy and chemotherapy, however, remains unknown [19].

Lastly, Leiomyosarcomas of the IVC present a significant technical challenge. They often involve adjacent organs, and because negative margins must be achieved, en bloc resection of involved structures is often required. Preoperative evaluation would typically include assessment of the IVC patency, site and degree of involvement, and extent of collaterals. If the vessel is patent and collaterals are not evident, reconstruction (usually with an interposition graft) should ideally be performed, especially if LMS involves trans- or suprarenal segments. If the vena cava is occluded prior to resection and/or clear collaterals are evidently developed, reconstruction does not need to be performed [20,21].

Simple ligation is possible after complete resection of the infra-renal IVC (segment I) and/or retrohepatic portion of the IVC (segment II) in association with resection of the right kidney. There is a difference in collateral circulation between the left and the right. Collaterals on the left (capsular, genital, renoazygo -lumbar veins) are generally sufficient for satisfactory venous return without occurrence of renal insufficiency. On the contrary, on the right there are no effective collaterals.

Some authors reported that reconstruction of the IVC is not necessary for resection of tumors below the level of the hepatic veins. Lower-extremity edema after ligation of the IVC is well tolerated. Acute renal failure can be a frequent, albeit transient, early postoperative complication [22].

\section{Conclusion}

Leiomyosarcomas are the most common malignancy involving the IVC. Although correlations between clinical manifestations and the location of the tumor within the IVC have been noted the more frequent use of CT scan in investigating patients with abdominal symptoms may aid in an earlier diagnosis and can be used for staging, planning treatment, and follow up. Chemotherapy and/or radiation therapy may serve as adjuncts to surgical resection but margin negative surgical resection is the main treatment modality that is expected to offer a chance of long-term survival.

\section{References}

1. Saptarshi Biswas, Arpit Amin, Suhaib Chaudry, Saju Joseph. Leiomyosarcoma of the Inferior Vena Cava - Radical Resection, Vascular Reconstruction and Challenges: A Case Report and Review of Relevant Literature. World Journal of Oncology. 2013;4(2):107-113.

2. Felix EL, Wood DK, Das Gupta TK. Tumors of the retroperitoneum. Curr Probl Cancer. 1981;6(1):1-47.

3. M. Hashimoto, T. Kobayashi, H. Tashiro, Amano H, Oshita A, Tanimoto $\mathrm{Y}$, et al. A huge metastatic liver tumor from leiomyosarcoma of the inferior vena cava: report of a case. Surgery Today. 2012;42(5): 505508. doi: 10.1007/s00595-011-0070-z.
4. Shindo S, Matsumoto H, Ogata K, Katahira S, Kojima A, Iyori K, et al. Surgical treatment of retroperitoneal leiomyosarcoma invading the inferior vena cava: report of three cases. Surg Today. 2002;32(10):929-933.

5. Kieffer E, Alaoui M, Piette JC, Cacoub P, Chiche L. Leiomyosarcoma of the inferior vena cava: experience in 22 cases. Ann Surg. 2006, 244(2):289-295.

6. Tameo MN, Calligaro KD, Antin L, Dougherty MJ. Primary leiomyosarcoma of the inferior vena cava: reports of infrarenal and suprahepatic caval involvement. J Vasc Surg. 2010;51(1):221-224. doi: $10.1016 /$ j.jvs.2009.07.111.

7. Anaya DA, Lev DC, Pollock RE. The role of surgical margin status in retroperitoneal sarcoma. J Surg Oncol. 2008;98(8):607-610. doi: $10.1002 /$ jso.21031.

8. Lewis JJ, Leung D, Woodruff JM, Brennan MF. Retroperitoneal softtissue sarcoma: analysis of 500 patients treated and followed at a single institution. Ann Surg. 1998;228(3):355-365.

9. Bonvalot S, Rivoire M, Castaing M, Stoeckle E, Le Cesne A, Blay JY, et al. Primary retroperitoneal sarcomas: a multivariate analysis of surgical factors associated with local control. J Clin Oncol. 2009;27(1):31-37. doi: 10.1200/JC0.2008.18.0802.

10. Gronchi A, Lo Vullo S, Fiore M, Mussi C, Stacchiotti S, Collini P, et al. Aggressive surgical policies in a retrospectively reviewed singleinstitution case series of retroperitoneal soft tissue sarcoma patients. J Clin Oncol. 2009;27(1):24-30. doi: 10.1200/JC0.2008.17.8871.

11.Zlotecki RA, Katz TS, Morris CG, Lind DS, Hochwald SN. Adjuvant radiation therapy for resectable retroperitoneal soft tissue sarcoma: the University of Florida experience. Am J Clin Oncol. 2005;28(3):310316 .

12. Pawlik TM, Pisters PW, Mikula L, Feig BW, Hunt KK, Cormier JN, et al. Long-term results of two prospective trials of preoperative external beam radiotherapy for localized intermediate- or high-grade retroperitoneal soft tissue sarcoma. Ann Surg Oncol. 2006;13(4):508517.

13. Gortzak E, Azzarelli A, Buesa J, Bramwell VH, van Coevorden F, van Geel AN, et al. E.O.R.T.C. Soft Tissue Bone Sarcoma Group and the National Cancer Institute of Canada Clinical Trials Group/Canadian Sarcoma Group: A randomised phase II study on neo-adjuvant chemotherapy for 'high-risk' adult soft-tissue sarcoma. Eur J Cancer. 2001;37(9):1096-1103.

14. Thomas DM, O'Sullivan B, Gronchi A. Current concepts and future perspectives in retroperitoneal soft-tissue sarcoma management. Expert Rev Anticancer Ther. 2009;9(8):1145-1157. doi: 10.1586/ era.09.77.

15. Mendenhall WM, Zlotecki RA, Hochwald SN, Hemming AW, Grobmyer SR, Cance WG. Retroperitoneal soft tissue sarcoma. Cancer. 2005;104(4):669-675.

16. Mingoli A, Cavallaro A, Sapienza P, Di Marzo L, Feldhaus RJ, Cavallari N. International registry of inferior vena cava leiomyosarcoma: analysis of a world series on 218 patients. Anticancer Res. 1996;16(5B):32013205.

17. Mingoli A, Feldhaus RJ, Cavallaro A, Stipa S. Leiomyosarcoma of the inferior vena cava: analysis and search of world literature on 141 patients and report of three new cases. J Vasc Surg. 1991;14(5):688699.

18. Sulpice L, Rayar M, Levi Sandri GB, de Wailly P, Henno S, Turner K. Leiomyosarcoma of the inferior vena cava. Journal of Visceral Surgery. 2016;153(3):161-165. doi: 10.1016/j.jviscsurg.2015.11.002. 
19. Ito H, Hornick JL, Bertagnolli MM, George S, Morgan JA, Baldini EH, et al. Leiomyosarcoma of the inferior vena cava: survival after aggressive management. Ann Surg Oncol. 2007;14(12):3534-3541.

20. Fiore M, Colombo C, Locati P, Berselli M, Radaelli S, Morosi C, et al. Surgical technique, morbidity, and outcome of primary retroperitoneal sarcoma involving inferior vena cava. Ann Surg Oncol. 2012;19(2):511-518. doi: 10.1245/s10434-011-1954-2.
21. Hines OJ, Nelson S, Quinones-Baldrich WJ, Eilber FR. Leiomyosarcoma of the inferior vena cava: prognosis and comparison with leiomyosarcoma of other anatomic sites. Cancer. 1999;85(5):10771083.

22. Daylami R, Amiri A, Goldsmith B, Troppmann C, Schneider PD, Khatri VP. Inferior Vena Cava Leiomyosarcoma: Is Reconstruction Necessary after Resection. J Am Coll Surg. 2010;210(2):185-190. doi: 10.1016/j. jamcollsurg.2009.10.010 